
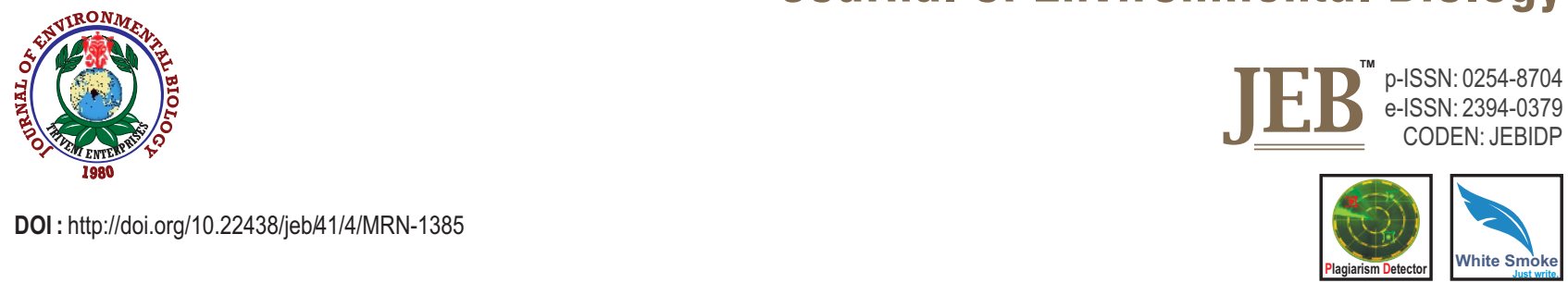

\title{
A preliminary study on bacterial composition in the subgingival plaque of woman with periodontitis during pregnancy and menopause
}

\author{
K.I. Han ${ }^{1,2}$, Y.R. Seo ${ }^{1}$, B.B. Patnaik ${ }^{3,4}$, H.J. Kwon ${ }^{1}$, E.G. Jung ${ }^{1}$, K.W. Nam ${ }^{1}$, W.J. Kim¹, J.S. Lee ${ }^{2}$ and M.D. Han ${ }^{1 *}$ \\ ${ }^{1}$ Department of Life Science and Biotechnology, College of Natural Sciences, Soonchunhyang University, Chungnam, 31538, Republic of Korea \\ ${ }^{2}$ Korean Collection for Type Cultures, Korea Research Institute of Bioscience and Biotechnology (KRIBB), Jeollabuk-do, 56212, Republic of Korea \\ ${ }^{3}$ Department of Biotechnology, Trident Academy of Technology, Bhubaneswar-751 024, India \\ ${ }^{4}$ PG Department of Biosciences and Biotechnology, Fakir Mohan University, Balasore-756 089, India \\ *Corresponding Author Email : mdhan@sch.ac.kr
}

\begin{abstract}
Aim: The aim of the present study was to explore the bacterial composition in subgingival plaque of females with periodontitis during pregnancy and
\end{abstract} menopause stages using $16 \mathrm{~S}$ ribosomal RNA(rRNA) gene pyrosequencing approach.

Methodology: Subgingival plaque was collected from four woman volunteers (healthy, periodontitis, periodontitis at pregnancy and periodontitis at menopause). The microbial community composition was analyzed by 454/Roche GS FLX chemistry pyrosequencing approach using the variable (V1V3) region of the $16 \mathrm{~S}$ rRNA gene. Pyrosequencing reads were sorted to get the clean reads that were annotated against the EzBioCloud data base for taxonomic classification. Operational Taxonomic Units (OTUs) were assigned and shared, and subsequently identified using CLCOMMUNITY software.

Results: Pyrosequencing yielded 13,939 sequences comprising of 13 phyla, 124 genera, and 372 species. The predominant microbial phyla in subgingival plaque of all woman volunteers included Firmicutes, Actinobacteria, Fusobacteria, Bacteroidetes, and Proteobacteria. In the healthy volunteer, Streptococcus (52.4\%) formed the predominant genus while in woman with periodontitis Streptococcus (24.6\%) and Fusobacterium (11.7\%) predominated. In the periodontitis volunteer with pregnancy, the predominant genus included Streptococcus $(25.8 \%)$ and Fusobacterium (22.4\%), whereas volunteer with menopause, the gingivitis was associated with genus Alloprevotella (19.5\%), Leptotrichia (14.3\%), Fusobacterium (12.3\%), and Porphyromonas (12.0\%).

Interpretation: This study proves on preliminary basis that the subgingival microbiome of woman with periodontitis at pregnancy or menopause tend to differ from that of healthy woman, and these species included certain periodontal pathogens such as Fusobacterium nucleatum and Porphyromonas gingivalis.

Key words: $16 \mathrm{~S}$ rRNA gene, Metagenomics, Oral microbiome, Periodontitis, Subgingival plaque

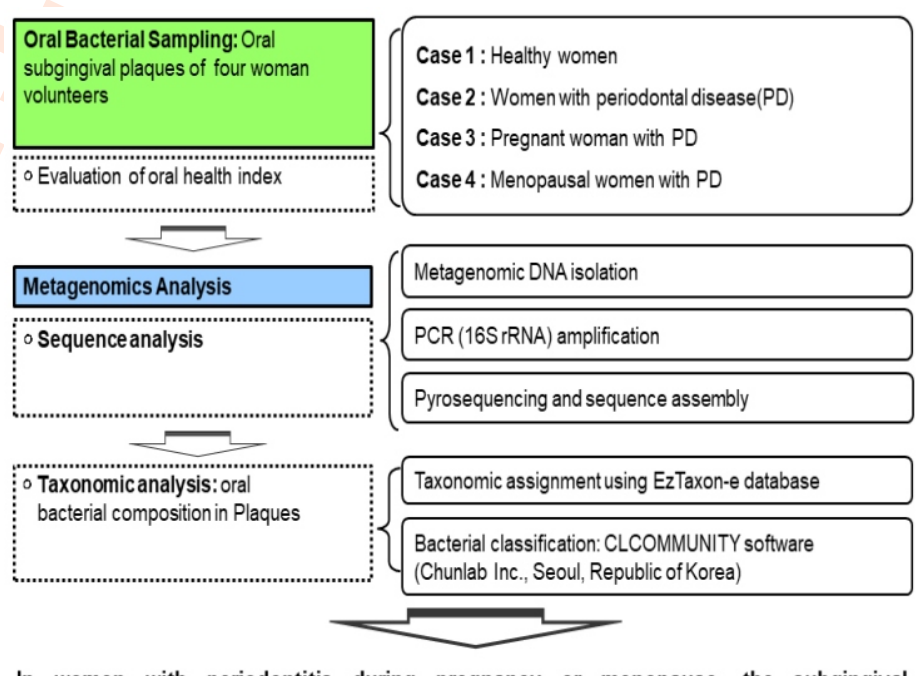

In women with periodontitis during pregnancy or menopause, the subgingival microbiome differed from that of healthy women, and these species included certain periodontal pathogens such as Fusobacterium nucleatum and Porphyromonas gingivalis.

How to cite : Han, K.I., Y.R. Seo, B.B. Patnaik, H.J. Kwon, E.G. Jung, K.W. Nam, W.J. Kim, J.S. Lee and M.D. Han: A preliminary study on bacterial composition in the subgingival plaque of woman with periodontitis during pregnancy and menopause. J. Environ. Biol., 41, 711-717(2020). 


\section{Introduction}

Periodontitis is an inflammatory condition that affects the gingiva, bone and other tissues that support the teeth. Periodontitis is responsible for tooth loss in adults, leading to nutritional disorders, reduced self-esteem, and socio-economic costs (Tonetti et al., 2017). Periodontitis is caused by the shifts in the composition of bacterial communities on intraoral surfaces, which promote inflammation (Abusleme et al., 2013). The pathogenesis of periodontitis is multi-factorial and may involve genetic and/or epigenetic factors (Luo et al., 2018). The hormonal changes that occur during puberty, menstruation, pregnancy and menopause can increase the sensitivity of gums to bacterial plaque. At puberty, variations in the level of female sex hormones, estrogen and progesterone, lead to softer gums that are more sensitive to bleeding. During monthly cycle (menses), some woman experience swollen gums, lesions, swollen salivary glands, or bleeding gums. Pregnancy is associated with gingivitis, a condition associated with red, puffy or tender gums attributed to increased progesterone levels (Jafri et al., 2015). Female-sex hormones impact the composition of oral microbiome, both qualitatively and quantitatively (Mariotti, 1994). This has been demonstrated in the gingiva; on periosteal fibers, scattered fibroblasts of the lamina propria, periodontal ligament fibroblasts, and osteoblasts (Mariotti, 1994). Further, during menopause, woman experience xerostomia (dry mouth), hyposalivation, and altered taste sensations, that influences the salivary microbiome to menopausal gingivostomatitis (Mahesh et al., 2014). According to a report of American Academy of Periodontology, women are $26 \%$ more likely to have periodontitis than men with higher incidence noted in pregnant woman. Cultivation, microscopy, DNA-DNA checkerboard and quantitative real-time PCR approaches have been used to evaluate the association of periodontitis condition in pregnant woman (Gürsoy et al., 2009). During pregnancy, subgingival periodontal pathogens such as Porphyromonas gingivalis and Prevotella intermedia promote inflammation. Moreover, the severity is correlated with maternal hormonal levels (Carrillo-de-Albornoz et al., 2010). Black-pigmented Bacteroides (BPB) are abundant in pregnant woman with gingivitis, although it is unclear whether this is due to inflammation or imbalances in hormone levels (Kumar et al., 2013).

High-throughput 16S rRNA sequencing facilitates an analysis of the relationships of the total bacterial diversity in the oral cavity (Park et al., 2015). Pyrosequencing of the 16 S rRNA amplicons from the subgingival space of periodontitis patients yielded $\sim 700$ species, including $P$. gingivalis and new species such as Filifactor alocis(Griffen et al., 2011). In smokers with periodontitis, Aggregatibacter actinomycetemcomitans and $P$. gingivalis are reportedly the predominant bacteria (Shchipkova et al., 2010). Further, bacteria at peri-implant and periodontal sites have been identified by amplification, sequencing and alignment of 16S rRNA genes (Schaumann et al., 2014). However, application of metagenomics for the analysis of microbial communities in the subgingival cavity of woman with periodontitis is limited. In this study, the subgingival microbiome of pregnant and menopausal woman volunteers with periodontitis were analyzed using $16 \mathrm{~S}$ rRNAgene pyrosequencing approach.

\section{Materials and Methods}

Subject selection: The study was approved by the Institutional Review Board of Soonchunhyang University (Approval number: 1040875-201409-BR-031). The woman volunteers (healthy, periodontitis, pregnant with periodontitis and menopausal with periodontitis) were recruited from the Soonchunhyang University Hospital, Asan, Republic of Korea. After obtaining affirmative consent from four woman volunteers, oral health status was evaluated by a dentist according to the Oral Health Survey Guidelines of World Health Organization (WHO) (Petersen and Baez, 2013). Oral health was evaluated considering three indexes. First, the Silness-Löe plaque index (PI) estimates the quantity of plaque in terms of tooth area covered. Second, the gingival index (GI) (Löe and Silness, 1963) assesses qualitative changes in the gingival condition, and third the periodontitis disease index (PDI) measures the periodontal status of an individual person (Ramfjord, 1967). All woman subjects showed signs of chronic periodontitis and had at least 24 teeth, including at least four molars. Further, it is noted that the periodontitis stage is more appropriate for examining the effect of female hormone on the subgingival microbiome than the gingivitis and advanced periodontitis stages (Table 1).

Sampling: Subgingival plaque samples were collected from right and left sides: 4 gingival sites (4 gingival sites/two teeth) from the first and second molar of mandible using Gracey curettes. Care was taken not to sample from infected sites such as deep caries, pulp necrosis, incomplete endodontic treatments, and secondary tooth decay. Further, samples were not taken from teeth with chronic apical periodontitis. The samples were lysed in $1 \mathrm{ml}$ of RNAzol solution and frozen at $70^{\circ} \mathrm{C}$ for later use.

DNA extraction, PCR amplification and Pyrosequencing: Bacterial DNA was extracted from oral plaque samples using Power Soil DNA isolation kit (MO BIO Laboratories, Carlsbad, $\mathrm{CA}$ ) according to the manufacturer's instructions. The purified DNA was quantified using NanoDrop ND-1000 Spectrophotometer (NanoDrop Technologies, Inc., Wilmington, DE) and analyzed on an Agilent 2100 Bioanalyser (Agilent Technologies, Santa Clara, CA).

PCR amplification was performed using primers targeting the V1 to V3 regions of $16 S$ rDNA: Universal primers 27F (5'CCTATCCCCTGTGTGCCTTGGCAGTC-TCAG-ACAGAGTTTGATCMTGGCTCAG-3' (underlining primer-target sequence) and 518R (5'-CCATCTCATCCCTGCGTGTCTCC GAC-TCAG-X-AC-CAGCAGCCGCGGTAATACG-3'); where ' $X$ ' indicates a unique barcode. PCR involved an initial denaturation of $95^{\circ} \mathrm{C}$ for $5 \mathrm{~min}$; followed by 30 cycles of denaturation at $95^{\circ} \mathrm{C}$ for 
Table 1: Clinical and demographic characteristics of four woman volunteers

\begin{tabular}{|c|c|c|c|c|c|}
\hline \multirow[t]{2}{*}{ Case } & \multirow[t]{2}{*}{ Woman participants } & \multirow[t]{2}{*}{ Age (year) } & \multicolumn{3}{|c|}{ Oral Health Index } \\
\hline & & & $\begin{array}{l}\text { Plaque Index } \\
(\mathrm{PI})^{\mathrm{a}}\end{array}$ & $\begin{array}{l}\text { Gingival Index } \\
(\mathrm{Gl})^{\mathrm{b}}\end{array}$ & $\begin{array}{l}\text { Periodontal Disease Index } \\
\text { (PDI) }^{c}\end{array}$ \\
\hline 1 & Healthy woman & 26 & 1 & 0 & 0 \\
\hline 2 & Woman with periodontal disease & 25 & 3 & 2 & 5 \\
\hline 3 & Pregnant woman with periodontal disease & 35 & 3 & 2 & 5 \\
\hline 4 & Menopausal woman with periodontal disease & 51 & 3 & 3 & 6 \\
\hline
\end{tabular}

${ }^{a}$ Plaque Index(PI): tooth is assigned a score of 0 to 3 (Löe and Silness, 1963). ${ }^{b}$ Gingival index (GI): marginal and interproximal tissues are separately scored from 0 to 3(Löe and Silness, 1963). 'Periodontal Disease Index(PDI): Six teeth are examined and scored. The higher the PDI score, greater the severity of periodontitis(Ramfjord, 1967)

$30 \mathrm{~s}$, annealing at $55^{\circ} \mathrm{C}$ for $30 \mathrm{~s}$, extension at $72^{\circ} \mathrm{C}$ for $30 \mathrm{~s}$ and a final elongation at $72^{\circ} \mathrm{C}$ for $5 \mathrm{~min}$. PCR products were resolved in $2 \%$ agarose gel electrophoresis and visualized using the Gel Doc system (BioRad, Hercules, CA). Amplicons were purified using a QIAquick PCR Purification Kit (Qiagen, Valencia, CA). Subsequently, equimolar concentrations of amplicons were prepared, clonally amplified on capture beads by emulsion PCR and the enriched DNA beads were deposited into a PicoTiter plate. Pyrosequencing was carried out at Chunlab Inc., (Seochogu, Seoul), using the GS Junior Sequencing System (Roche, Branford, CT) according to the manufacturer's instructions.

Bioinformatics analysis: The obtained reads were sorted according to their barcodes. The barcode, linker, and primer sequences were trimmed from the original sequencing reads. Further, reads containing ambiguous nucleotides, those with low quality scores (average < 25), and reads shorter than 300 bp were discarded from the analysis. Potential chimera sequences were detected by the Bellerophon method and removed from the analysis. We used the EzBioCloud Database (https://www. ezbiocloud.net) for taxonomic classification of reads (Yoon et al., 2017).

The richness and diversity of samples were determined by abundance-based coverage estimation and by calculating the Shannon diversity index at $3 \%$ distance. A revised complete sequence dataset was clustered and assigned to Operational Taxonomic Units (OTUs). CLCOMMUNITY software (Chunlab Inc., Seoul, Republic of Korea) was used to identify shared OTUs for comparison purposes. The species, genus, family and phylum levels were defined at $3 \%, 5 \%, 10 \%$ and $20 \%$ dissimilarities, respectively (Schloss and Handelsman, 2005).For taxonomybased analysis, ARB-SILVA database 114 was used with an $80 \%$ confidence threshold (Quast et al., 2013). Rarefaction curves and richness estimates were analyzed using R VEGAN (Oksanen et al., 2013) and MOTHUR (Schloss et al., 2009) packages, respectively.

\section{Results and Discussion}

The oral microbiome is an indicator of total body wellness and is influenced by factors such as sex hormone, diet, temperature and $\mathrm{pH}$. The oral microbial communities can modulate the outcome of diseases of other organs, e.g., association between periodontitis and lung diseases (Raghavendran et al., 2007), and cancer (Sabbah et al., 2019). Periodontitis (gingivitis) is an inflammatory condition of the gums that presents initially as bleeding gums, followed by plaque formation. The succession of bacterial communities during different phases of woman's growth (including puberty) has been reported (Contreras et al., 2006). However bacterial composition of subgingival plaque in females with periodontitis during pregnancy and menopause stages is not clear.

As shown in Table 2, a total of 14,048 reads passed quality control. The sequence comprised $\sim 17,900$ amplicons from V1 to V3 hypervariable regions of the $16 \mathrm{~S}$ rRNA gene. Most of the high-quality reads showed $97 \%$ nucleotide similarity, indicating species - level phylotypes. The $3 \%$ sequence dissimilarity cut-off value is used in most microbial dynamics and diversity studies to profile species-level phylotypes. The reads showing a greater sequence variation were determined at the genus, family and order levels. The greatest proportion of reads with species-level phylotypes was registered from the sequence of healthy woman volunteer, followed by periodontitis, periodontitis and pregnant and periodontitis and menopausal subjects. Pyrosequencing identified 13 phyla, 124 genera and 372 species. The bacterial communities identified belonged to phyla Firmicutes, Bacterioidetes, Fusobacteria, Proteobacteria, Actinobacteria and Spirochaetes. Firmicutes predominated in the healthy and periodontitis subjects and Bacterioidetes in the pregnant and menopausal woman volunteer with periodontitis. Fusobacterium was also detected in abundance in all the woman volunteers. These data suggest a shift in the bacterial community under periodontitis condition from Firmicutes and Proteobacteria to Bacterioidetes and Fusobacteria. Spirochaetes were also detected in the pregnant and menopausal woman volunteers with periodontitis. At genus-level (Table 3), Streptococcus was the dominant bacteria in healthy woman $(50.5 \%)$ and women with periodontal disease (25.9\%). In pregnant and menopausal periodontitis woman, the dominant genera were Alloprevotella and Prevotella, respectively. 
Table 2 : Sequencing information and taxonomic assignments of plaque bacterial communities

\begin{tabular}{llllll}
\hline Classification & Similarity range & \multicolumn{4}{c}{ No. of reads (\%) } \\
\cline { 3 - 6 } & & Healthy woman & Periodontitis woman & Pregnant Woman & Menopausal woman \\
\hline Species & $x>=97 \%$ & $4920(98.73)$ & $3233(96.50)$ & $2550(95.93)$ & $2895(94.70)$ \\
Genus & $97>x>=94.5 \%$ & $52(1.04)$ & $93(2.77)$ & $79(2.97)$ & $96(3.14)$ \\
Family & $94.5>x>=86.5 \%$ & $10(0.20)$ & $22(0.65)$ & $27(1.01)$ & $50(1.63)$ \\
Order & $86.5>x>=82 \%$ & $1(0.02)$ & $0(0.00)$ & $2(0.07)$ & $14(0.45)$ \\
Class & $82>x>=78.5 \%$ & $0(0.00)$ & $2(0.05)$ & $0(0.00)$ & $2(0.06)$ \\
Phylum & $78.5>x>=75 \%$ & $0(0.00)$ & $0(0.00)$ & $0(0.00)$ & $0(0.00)$ \\
Unidentified & $75 \%>x$ & $0(0.00)$ & $0(0.00)$ & $0(0.00)$ & $0(0.00)$ \\
Total & 14,048 reads & $4983(100)$ & $3350(100)$ & $2658(100)$ & $3057(100)$ \\
\hline
\end{tabular}

Table 3 : Relative genus-level bacterial composition in the oral plaque

\begin{tabular}{|c|c|c|c|c|c|c|c|c|c|c|c|c|}
\hline \multirow[t]{2}{*}{$\begin{array}{l}\text { Rank } \\
\text { of } \\
\text { genus }\end{array}$} & \multicolumn{3}{|l|}{$\begin{array}{l}\text { Healthy women } \\
\text { ( } 26 \text { years old) }\end{array}$} & \multicolumn{3}{|c|}{$\begin{array}{l}\text { Women with periodontal } \\
\text { disease ( } 25 \text { year old })\end{array}$} & \multicolumn{3}{|l|}{$\begin{array}{l}\text { Pregnant woman with } \\
\text { periodontal disease } \\
\text { ( } 35 \text { year old) }\end{array}$} & \multicolumn{3}{|c|}{$\begin{array}{l}\text { Menopausal women with } \\
\text { periodontal disease } \\
\text { (51 year old) }\end{array}$} \\
\hline & Genus name & $\%$ & No. & Genus name & $\%$ & No. & Genus name & $\%$ & No. & Genus name & $\%$ & No. \\
\hline 1 & Streptococcus & 52.4 & 2612 & Streptococcus & 25.9 & 866 & Alloprevotella & 19.5 & 518 & Prevotella & 25.4 & 776 \\
\hline 2 & Haemophilus & 15.6 & 779 & Fusobacterium & 22.4 & 749 & Leptotrichia & 14.3 & 379 & Fusobacterium & 13.9 & 426 \\
\hline 3 & Veillonella & 14.0 & 697 & Prevotella & 6.6 & 222 & Fusobacterium & 12.3 & 326 & Streptococcus & 9.4 & 287 \\
\hline 4 & Actinomyces & 5.0 & 248 & Parvimonas & 4.5 & 149 & Porphyromonas & 12.0 & 319 & Atopobium & 6.6 & 203 \\
\hline 5 & Lautropia & 3.8 & 188 & Alloprevotella & 4.2 & 141 & Treponema & 9.6 & 256 & Treponema & 6.5 & 198 \\
\hline 6 & Rothia & 2.9 & 146 & Treponema_g1 & 4.0 & 134 & Treponema_g1 & 4.2 & 111 & Dialister & 3.6 & 110 \\
\hline 7 & Fusobacterium & 1.9 & 95 & Actinomyces & 3.2 & 108 & Peptostreptococcus & 3.1 & 81 & Shuttleworthia & 3.1 & 94 \\
\hline 8 & Prevotella & 1.2 & 59 & Leptotrichia & 2.9 & 96 & Prevotella & 2.7 & 71 & Megasphaera & 2.4 & 74 \\
\hline 9 & Neisseria & 0.8 & 41 & Selenomonas_g1 & 2.7 & 90 & Mycoplasma_g4 & 2.6 & 70 & Alloprevotella & 2.0 & 61 \\
\hline 10 & Capnocytophaga & 0.7 & 35 & Fretibacterium & 1.7 & 58 & Selenomonas & 2.1 & 57 & Olsenella & 1.8 & 54 \\
\hline 11 & Aggregatibacter & 0.2 & 11 & Corynebacterium & 1.6 & 54 & Catonella & 2.1 & 56 & Eubacterium_g10 & 1.7 & 51 \\
\hline 12 & Campylobacter & 0.2 & 9 & Campylobacter & 1.6 & 52 & Fretibacterium & 1.8 & 49 & Porphyromonas & 1.6 & 50 \\
\hline 13 & Kingella & 0.1 & 7 & Treponema & 1.4 & 47 & Johnsonella & 1.8 & 47 & Treponema_g1 & 1.5 & 46 \\
\hline 14 & Cardiobacterium & 0.1 & 6 & Saccharimonas & 1.4 & 46 & Selenomonas_g1 & 1.7 & 45 & Eggerthia & 1.3 & 39 \\
\hline 15 & Corynebacterium & 0.1 & 5 & AF432141_g & 1.3 & 43 & Streptococcus & 0.9 & 25 & Parvimonas & 1.2 & 36 \\
\hline 16 & Granulicatella & 0.1 & 5 & Johnsonella & 1.2 & 41 & Parvimonas & 0.9 & 24 & Fretibacterium & 1.1 & 34 \\
\hline 17 & Actinobaculum & 0.1 & 5 & Veillonella & 1.0 & 34 & Phocaeicola & 0.7 & 19 & Cryptobacterium & 1.0 & 30 \\
\hline 18 & Leptotrichia & 0.08 & 4 & Gemella & 1.0 & 34 & GQ422727_g & 0.7 & 19 & Campylobacter & 0.9 & 28 \\
\hline 19 & Veillonellaceae_uc & 0.08 & 4 & Aggregatibacter & 1.0 & 33 & Actinomyces & 0.6 & 17 & Actinomyces & 0.9 & 27 \\
\hline 20 & ETC & 0.54 & 27 & ETC & 10.5 & 353 & ETC & 6.4 & 169 & ETC & 14.2 & 433 \\
\hline
\end{tabular}

In healthy volunteer, Streptococcus pseudopneumoniae predominated, followed by Haemophilus parainfluenzae, Streptococcus sanguinis and Veillonella dispar. Streptococcus sp. predominated in the plaque microbiome of healthy woman volunteer; the species detected were $S$. gordonii, S. tigurinus, $S$. mitis and $S$. dentisani. In the periodontitis woman volunteer, Fusobacterium nucleatum and S. pseudopneumoniae were the predominant species (Table 4). Further, the other Fusobacterium and Streptococcus species that were represented in the subgingival plaque of periodontitis woman volunteer included $S$. cristatus, F. periodonticum, F. canifelinum, S. oralis and S. mitis (data not shown). Alloprevotella tannerae and F. nucleatum also predominated in the pregnant and menopausal woman volunteers with periodontitis, respectively, followed by $P$. gingivalis and Prevotella multiformis, respectively. The bacterial communities at genus and species levels in the pregnant and menopausal woman volunteers with periodontitis disease were considered closer to the bacterial community in the woman subject with periodontitis. Further, the abundance of Veillonella and Streptococcus is associated with health-associated biofilms (Bernardi et al., 2020; Sanz et al., 2017; Naginyte et al., 2019). In recent studies, $P$. gingivalis (Bacterioidetes), Treponemas denticola (Spirochaetes) and F. nucleatum (Fusobacteria) were major bacterial species causing periodontal disease(PérezChaparro et al., 2014; Choi et al., 2018). In our study, while F. nucleatum predominated in woman volunteers with periodontitis, $P$. gingivalis was detected only in pregnant woman volunteers with periodontitis (Table 4). F. nucleatum is a species involved in 
Table 4 : Relative species-level bacterial composition in the oral plaque.

\begin{tabular}{|c|c|c|c|c|c|c|c|c|}
\hline $\begin{array}{l}\text { Rank } \\
\text { of } \\
\text { species }\end{array}$ & $\begin{array}{l}\text { Healthy woman } \\
\text { ( } 26 \text { year old) }\end{array}$ & $\%$ & $\begin{array}{l}\text { Woman with periodontal } \\
\text { disease ( } 25 \text { year old) }\end{array}$ & $\%$ & $\begin{array}{l}\text { Pregnant woman with } \\
\text { periodontal disease } \\
\text { ( } 35 \text { year old) }\end{array}$ & $\%$ & $\begin{array}{l}\text { Menopausal woman } \\
\text { with periodontal disease } \\
\text { ( } 51 \text { year old) }\end{array}$ & $\%$ \\
\hline 1 & $\begin{array}{l}\text { Streptococcus } \\
\text { pseudopneumoniae }\end{array}$ & 21.3 & $\begin{array}{l}\text { Fusobacterium } \\
\text { nucleatum }\end{array}$ & 19.0 & $\begin{array}{l}\text { v Alloprevotella } \\
\text { tannerae }\end{array}$ & 18.8 & $\begin{array}{l}\text { Fusobacterium } \\
\text { nucleatum }\end{array}$ & 13.6 \\
\hline 2 & $\begin{array}{l}\text { Haemophilus } \\
\text { parainfluenzae }\end{array}$ & 15.3 & $\begin{array}{l}\text { Streptococcus } \\
\text { pseudopneumoniae }\end{array}$ & 13.0 & $\begin{array}{l}\text { Porphyromonas } \\
\text { gingivalis }\end{array}$ & 12.0 & Prevotella multiformis & 7.8 \\
\hline 3 & Streptococcus sanguinis & 13.1 & Parvimonas micron & 4.5 & $\begin{array}{l}\text { Fusobacterium } \\
\text { nucleatum }\end{array}$ & 11.4 & Streptococcus anginosus & 6.3 \\
\hline 4 & Veillonella dispar & 10.0 & Alloprevotella tannerae & 3.9 & AY134896_s & 10.6 & Atopobium rimae & 6.3 \\
\hline 5 & Streptococcus gordonii & 6.5 & Streptococcus cristatus & 3.4 & $\begin{array}{l}\text { Peptostreptococcus } \\
\text { stomatis }\end{array}$ & 3.0 & Prevotella nigrescens & 5.9 \\
\hline 6 & Streptococcus tigurinus & 5.6 & Actinomyces cardiffensis & 2.6 & Mycoplasma faucium & 2.6 & Shuttleworthia satelles & 3.1 \\
\hline 7 & Lautropia mirabilis & 3.8 & Treponema socranskii & 2.0 & JN713365_s & 2.2 & Dialisterinvisus & 2.6 \\
\hline 8 & Streptococcus mitis & 2.5 & Prevotella intermedia & 1.8 & Leptotrichia wadei & 2.0 & Prevotella intermedia & 2.16 \\
\hline 9 & Veillonella parvula & 2.4 & $\begin{array}{l}\text { Fusobacterium } \\
\text { periodonticum }\end{array}$ & 1.7 & 4P000005_s & 1.8 & AF385554_S & 1.9 \\
\hline 10 & viscosus & 2.3 & Prevotella baroniae & 1.7 & AF287774_s & 1.8 & Alloprevotella tannerae & 1.9 \\
\hline 11 & Streptococcus dentisani & 1.5 & $\begin{array}{l}\text { Corynebacterium } \\
\text { matruchotii }\end{array}$ & 1.5 & $\begin{array}{l}\text { Fretibacterium } \\
\text { fastidiosum }\end{array}$ & 1.8 & $\begin{array}{l}\text { Anaeroglobus } \\
\text { geminatus }\end{array}$ & 1.8 \\
\hline 12 & Rothia dentocariosa & 1.5 & Fusobacterium canifelinum & 1.4 & Catonellamorbi & 1.7 & Eubacterium infirmum & 1.6 \\
\hline 13 & Fusobacterium nucleatum & 1.5 & AY349371_s & 1.4 & Selenomonas sputigena & 1.7 & Treponema medium & 1.6 \\
\hline 14 & Rothia aeria & 1.3 & Streptococcus oralis & 1.3 & Treponema medium & 1.6 & Treponema denticola & 1.5 \\
\hline 15 & Actinomyces oris & 1.2 & Streptococcus mitis & 1.3 & Treponema socranskii & 1.2 & Eggerthia catenaformis & 1.3 \\
\hline 16 & Etc & 10.0 & Etc & 39.5 & Etc & 25.7 & Etc & 40.8 \\
\hline Total & & 100 & & 100 & & 100 & & 100 \\
\hline
\end{tabular}

dental plaque development and is found in the oral tissue of woman with normal or high-risk pregnancies (Tateishi et al., 2012). It is also closely associated with periodontal attachment loss and has local immunosuppressive activity (Silva et al., 2015). Similarly, $P$. gingivalis is abundant in gingival sulcus during early and middle stages of pregnancy (Fujiwara et al., 2017). This bacterium modulates the host inflammatory response directly or indirectly and destroys periodontal tissue (How et al., 2016; Fujiwara et al., 2017). Subject with periodontitis had greater taxonomic diversity than healthy subjects in agreement with a prior study (Griffen et al., 2012).

Next-generation sequencing technologies facilitate analysis of microbial diversity. 16S rRNA pyrosequencing was utilized to profile the subgingival microbiome of woman with periodontitis. 454 pyrosequencing has been applied to profile the bacterial communities in throat, stomach and fecal samples of humans (Andersson et al., 2008), and soil sediments samples (Pramanik et al., 2015). All taxonomic classifications were assigned using Bayesian algorithm developed for Ribosomal Database Project (RDP) classifier and Ez Bio Cloud database. Pyrosequencing reads were utilized using the Ez Bio Cloud database which contains the phylotypes of cultured and uncultured microorganisms. A total of 195-300 OTUs per sample were detected in the periodontitis subject. OTUs are clustered 16S rRNA sequences and are used as proxies for species classification (Choi et al., 2018). Typically, 97\% and 95\% sequence identities are cut-offs for species and genera, respectively (de Gannes et al., 2013), including in oral biofilms (Pushalkar et al., 2011). But this is an approximate measure as two different species may have identical 16S rRNA sequences and/or a single species may have two copies of 16S rRNA gene that could differ by more than $97 \%$. In this study, $94 \%$ of pyrosequencing reads were classified as species. The sequences were taxonomically classified by comparison with the SILVA database (at a threshold of $97 \%$ identity).

The total plaque bacterial community richness was estimated by rarefaction. To determine richness and diversity, OTUs were identified at genetic distances of $3 \%$. In this study, the maximum 300 and 389.31 OTUs were observed and estimated in the woman subject with periodontitis condition. The ecological organization of communities was evaluated by calculating Shannon's diversity and Simpson's evenness indices. Shannon index values were $3.63,4.32$ and 4.35 in healthy, pregnant and periodontitis woman, respectively. The Simpson's index values were $0.053,0.036$ and 0.047 in the healthy, periodontitis, and pregnant woman volunteers with periodontitis, respectively (Table 5). Thus, bacterial diversity was higher in the volunteers with periodontitis compared to healthy volunteer. In subgingival plaques of healthy humans, four bacterial species dominated $(60 \%$ of the total bacteria) whereas woman with periodontal 
Table 5 : Species richness, diversity and evenness of oral plaque. Estimated statistical values obtained from the Mothur program

\begin{tabular}{|c|c|c|c|c|c|c|}
\hline \multirow[t]{2}{*}{ Case } & \multicolumn{2}{|c|}{ Read length (bp) } & \multirow[t]{2}{*}{ Observed"OTUs } & \multirow{2}{*}{$\begin{array}{l}\text { Estimated" } \\
\text { OTUs(Chao 1) }\end{array}$} & \multirow{2}{*}{$\begin{array}{l}\text { Simpson } \\
\text { Evenness }\end{array}$} & \multirow{2}{*}{$\begin{array}{l}\text { Shannon diversity } \\
\text { index }\end{array}$} \\
\hline & Mean & Maximum & & & & \\
\hline Healthy woman & 439.03 & 511 & 148 & 179.32 & 0.053 & 3.63 \\
\hline Periodontitis woman & 449.11 & 511 & 300 & 389.31 & 0.036 & 4.35 \\
\hline Pregnant Woman & 438.9 & 513 & 258 & 370.86 & 0.047 & 4.32 \\
\hline
\end{tabular}

*OTUs: Operational Taxonomic Unit

disease composed of 15 bacterial species (Table 4). This diversity of bacterial composition is for the following reasons: the microbial communities of oral cavity are in symbiosis with the host. However, in the presence of stressors, such as imbalance of hormone levels that can perturb this homeostasis, several oral infectious diseases may appear, including periodontitis (Sanz et al., 2017). In healthy individuals, the oral bacterial microbiome encompasses approximately 700 commonly occurring phylotypes, approximately half of which can be present at any time in any individual (Gao et al., 2018). These bacterial compositions of pregnancy and menopause women can be dynamic and adapt to a wide range of environments and interactions with other microbial species. Several evidences have been found that influence microbial communities in postmenopausal women, such as decrease of estrogen receptorbeta in oral mucosa, exfoliation of age-related normal buccal mucosa and increase in surface keratinization and lipid lamellae in the epithelial samples (Donald et al., 2013). In pregnancy, the literature describes at least some reasons that may contribute to the exacerbation of periodontal inflammation in the presence of high levels of estradiol and progesterone (Massoni et al., 2019). Some reasons are the vasodilatory effect of estrogen, suppression of the immune system, and quantitative and qualitative alterations of bacterial flora(Carrillo-de-Albornoz et al., 2010).

The results of this preliminary study suggest that the subgingival microbial communities in woman with periodontitis during pregnancy or menopause tend to differ from that of healthy woman, and these species included certain periodontal pathogens $F$. nucleatum and $P$. gingivalis. The study of microbial community dynamics in women with periodontitis would be crucial to establish pathogenesis of periodontal diseases correlating it to pregnancy and menopausal stages. Additionally, the microbial species can act as biomarkers of hormonal changes in women.

\section{Acknowledgments}

This study was supported in part by Soonchunhyang University Research Fund. The authors thank Dr. KK Kim who had collected oral plaque sample for this study from the Department of Dentistry, Soonchunhyang University Cheonan Hospital.

\section{References}

Abusleme, L., A.K. Dupuy, N. Dutzan, N. Silva, J.A. Burleson, L.D. Strausbaugh, J. Gamonal and P.I. Diaz: The subgingival microbiome in health and periodontitis and its relationship with community biomass and inflammation. ISME J., 7, 1016-1025 (2013).

Andersson, A.F., M. Lindberg, H. Jakobsson, F. Bäckhed, P. Nyrén and L. Engstrand: Comparative analysis of human gut microbiota by barcoded sequencing. PLOS ONE, 3, e2836 (2008).

Bernardi, S., L. Karygianni, A. Filippi, A.C. Anderson, A. Zürcher, E. Hellwig, K. Vach, G. Macchiarelli and A. Al-Ahmad: Combining culture and culture-independent methods reveals new microbial composition of halitosis patients' tongue biofilm. Microbiology Open, 9 , e958 (2020).

Carrillo-de-Albornoz, A., E. Figuero, D. Herrera and A. BasconesMartínez: Gingival changes during pregnancy: II. Influence of hormonal variations on the subgingival biofilm. J. Clin. Periodontol., 37, 230-240 (2010).

Choi, H., E. Kim, J. Kang, H.J. Kim, J.Y. Lee, J. Choi and J.Y. Joo: Realtime PCR quantification of 9 periodontal pathogens in saliva samples from periodontally healthy Korean young adults. J. Periodontal. Implant. Sci., 48, 261-271 (2018).

Choi, J. E., S.H. Choi, J.S. Lee, K.C. Lee, S.M. Kang, J.I. Kim, M.S. Choi, H.G. Kim, W.T. Seo, K.Y. Lee, B.C. Moon and Y. M. Kang: Analysis of microbial communities in local cultivars of astringent persimmon (Diospyros kaki) fruits grown in Gyeongnam Province of Korea. J. Environ. Biol., 39, 237-246 (2018).

Contreras, A., J.A. Herrera, J.E. Soto, R.M. Arce, A. Jaramillo and J.E. Botero: Periodontitis is associated with preeclampsia in pregnant woman. J. Periodontol., 77, 182-188 (2006).

de Gannes, V., G. Eudoxie and W.J. Hickey. Prokaryotic successions and diversity in composts as revealed by 454-pyrosequencing. Bioresour. Technol., 133, 573-580 (2013).

Donald, P.M., R. George, G. Sriram, B. Kavitha and B. Sivapathasundharam: Hormonal changes in exfoliated normal buccal mucosal cells. J. Cytol., 30, 252-256 (2013).

Fujiwara, N., K. Tsuruda, Y. Iwamoto, F. Kato, T. Odaki, N. Yamane, Y. Hori, Y. Harashima, A. Sakoda, A. Tagaya, H. Komatsuzawa, M. Sugai and M. Noguchi: Significant increase of oral bacteria in the early pregnancy period in Japanese women. J. Investig. Clin. Dent., 8, e12189 (2017).

Gao, L., G. Huang, S. Jiang, Y. Gu and F. Chen: Oral microbiomes: More and more importance in oral cavity and whole body. Protein Cell, 9 , 488-500 (2018).

Griffen, A.L., C.J. Beall, N.D. Firestone, E.L. Gross, J.M. Difranco, J.H. Hardman, B. Vriesendorp, R.A. Faust, D.A. Janies and E.J. Leys: CORE: A phylogenetically-curated $16 \mathrm{~S}$ rDNA database of the core 
oral microbiome. PLOS ONE,6, e19051 (2011).

Griffen, A.L., C.J. Beall, J.H. Campbell, N.D. Firestone, P.S. Kumar, Z.K. Yang, M. Podar and E.J. Leys: Distinct and complex bacterial profiles in human periodontitis and health revealed by $16 \mathrm{~S}$ pyrosequencing. ISME J, 6, 1176-1185(2012).

Gürsoy, M., G. Haraldsson, M. Hyvönen, T. Sorsa, R. Pajukanta and E. Könönen: Does the frequency of Prevotella intermedia increase during pregnancy? Oral. Microbiol. Immunol., 24, 299-303 (2009).

How, K.Y., K.P. Song and K.G. Chan. Porphyromonas gingivalis: An overview of periodontopathic pathogens below the gum line. Front. Microbiol., 7, 53 (2016).

Jafri, Z., A. Bhardwaj, M. Sawai and N. Sultan: Influence of female sex hormones on periodontium: A case series. J. Nat. Sci. Biol. Med., 6, S146-149 (2015)

Kumar, A., M. Basra, N. Begum, V. Rani, S. Prasad, A.K. Lamba, M. Verma, S. Agarwal and S. Sharma: Association of maternal periodontal health with adverse pregnancy outcome. J. Obstet. Gynaecol. Res., 39, 40-45 (2013).

Löe, H. and J. Silness: Periodontal disease in pregnancy. Acta. Odontol. Scand., 21,533-551 (1963).

Luo, Y., X. Peng, D. Duan, C. Liu, X. Xu and X. Zhou: Epigenetic regulations in the pathogenesis of periodontitis. Curr. Stem Cell Res. Ther., 13, 144-150 (2018).

Mahesh, D.R., G. Komali, K. Jayanthi, D. Dinesh, T.V. Saikavitha and P. Dinesh: Evaluation of salivary flow rate, $\mathrm{pH}$ and buffer in pre, post and post-menopausal women in HRT. J. Clin. Diagn. Res., 8, 233236 (2014).

Massoni, R.S.S., A.M.F. Aranha, F.Z. Matos, O.A. Guedes, Á.H. Borges, M. Miotto and A.N. Porto: Correlation of periodontal and microbiological evaluations, with serum levels of estradiol and progesterone, during different trimesters of gestation. Sci. Rep.,9, 11762 (2019).

Mariotti, A.: Sex steroid hormones and cell dynamics in the periodontium. Crit. Rev. Oral. Biol. Med.,5, 27-53 (1994).

Naginyte, M., T. Do, J. Meade, D.A. Devine and P.D. Marsh: Enrichment of periodontal pathogens from the biofilms of healthy adults. Sci. Rep., 9, 5491 (2019).

Oksanen, J., F.G. Blanchet, R. Kindt, P. Legendre, D. McGlinn, P.R. Minchin, R.B. O'Hara, G.L. Simpson, P. Solymos, M.H.H. Stevens, E. Szoecs and H. Wagner: Vegan: Community Ecology Package. R Package version 2.0-7 ed. (2013).

Park, O.J., H. Yi, J.H. Jeon, S.S. Kang, K.T. Koo, K.Y. Kum, J. Chun, C.H. Yun and S.H. Han: Pyrosequencing analysis of subgingival microbiota in distinct periodontal conditions. J. Dent. Res., 94, 921-927 (2015)

Petersen, P.E. and R.J. Baez: Oral health surveys: Basic methods. $5^{\text {th }}$ Edn., France: World Health Organization (2013).

Pérez-Chaparro, P.J., C. Gonçalves, L.C. Figueiredo, M. Faveri, E. Lobão, N. Tamashiro, P. Duarte and M. Feres: Newly identified pathogens associated with periodontitis: A systematic review. J. Dent. Res., 93, 846-858 (2014).

Pramanik, A., P. Basak, S. Banerjee, S. Sengupta, D. Chattopadhyay and M. Bhattacharyya: Pyrosequencing based profiling of the bacterial community in the Chilika Lake, the largest lagoon of India. Genom. Data, 4, 112-114 (2015)

Pushalkar, S., S.P. Mane, X. Ji, Y. Li, C. Evans, O.R. Crasta, D. Morse, R. Meagher, A. Singh and D. Saxena: Microbial diversity in saliva of oral squamous cell carcinoma. FEMS. Immunol. Med. Microbiol., 61, 269-277 (2011)

Quast, C., E. Pruesse, P. Yilmaz, J. Gerken, T. Schweer, P. Yarza, J. Peplies and F.O. Glöckner: The SILVA ribosomal RNA gene database project: Improved data processing and web-based tools. NucleicAcids Res., 41, D590-596 (2013).

Raghavendran, K., J.M. Mylotte and F.A. Scannapieco: Nursing homeassociated pneumonia, hospital-acquired pneumonia and ventilator-associated pneumonia: The contribution of dental biofilms and periodontal inflammation. Periodontol 2000, 44, 16477 (2007).

Ramfjord, S.P.: The Periodontal Disease Index (PDI). J. Periodontol., 38, 602-610 (1967).

Sabbah, W., M.O. Folayan and M.E. Tantawi: The link between oral and general health. Int. J. Dent., 2019, 1-2 (2019).

Sanz, M., D. Beighton, M.A. Curtis, J.A. Cury, I. Dige, H. Dommisch, R. Ellwood, R.A. Giacaman, D. Herrera, M.C. Herzberg, E. Könönen, P.D. Marsh, J. Meyle, A. Mira, A. Molina, A. Mombelli, M. Quirynen, E.C. Reynolds, L. Shapira and E. Zaura: Role of microbial biofilms in the maintenance of oral health and in the development of dental caries and periodontal diseases. Consensus Report of Group 1 of the Joint EFP/ORCA Workshop on the Boundaries Between Caries and Periodontal Disease. J. Clin. Periodontol., 44, S5-S11 (2017).

Schaumann, S., I. Staufenbiel, R. Scherer, M. Schilhabel, A. Winkel, S.N. Stumpp, J. Eberhard and M. Stiesch: Pyrosequencing of supraand subgingival biofilms from inflamed peri-implant and periodontal sites. BMC Oral Hlth., 14, 157 (2014).

Schloss, P.D. and J. Handelsman: Introducing DOTUR, a computer program for defining operational taxonomic units and estimating species richness. Appl. Environ. Microbiol., 71, 1501-1506 (2005).

Schloss, P.D., S.L. Westcott, T. Ryabin. J.R. Hall, M. Hartmann, E.B. Hollister, R.A. Lesniewski, B.B. Oakley, D.H. Parks, C.J. Robinson, J.W. Sahl, B. Stres, G.G. Thallinger, D.J. Van Horn and C.F. Weber: Introducing mothur: Open-Source, Platform-Independent, Community-Supported Software describing and comparing microbial communities. Appl. Environ. Microbiol., 75, 7537-7541 (2009).

Shchipkova, A.Y., H.N. Nagaraja and P.S. Kumar: Subgingival microbial profiles of smokers with periodontitis. J. Dent. Res., 89, 1247-1253 (2010).

Silva, N., L. Abusleme, D. Bravo, N. Dutzan, J. Garcia-Sesnich, R. Vernal, M. Hernández and J. Gamonal: Host response mechanisms in periodontal diseases. J. Appl. Oral. Sci., 23, 329$355(2015)$

Tateishi, F., K. Hasegawa-Nakamura, T. Nakamura, Y. Oogai, H. Komatsuzawa, K. Kawamata, T. Douchi, M. Hatae and K. Noguchi: Detection of Fusobacterium nucleatum in chorionic tissues of highrisk pregnant women. J. Clin. Periodontol., 39, 417-424 (2012).

Tonetti, M.S., S. Jepsen, L. Jin and J. Otomo-Corgel: Impact of the global burden of periodontal diseases on health, nutrition and wellbeing of mankind: A call for global action. J. Clin. Periodontol., 44, 456462(2017).

Yoon, S.H., S.M. Ha, S. Kwon, J. Lim, Y. Kim, H. Seo and J. Chun: Introducing EzBio Cloud: A taxonomically united database of $16 \mathrm{~S}$ rRNA gene sequences and whole-genome assemblies. Int. J. Syst. Evol. Microbiol., 67, 1613-1617 (2017). 